\title{
Reflexiones en torno al vínculo género-educación superior
}

\section{Reflections on the Gender-Higher Education Bond}

\author{
María del Carmen Acuña-Rodríguez ${ }^{1}$ \\ Universidad de Costa Rica \\ Centro de Evaluación Académica \\ San José, Costa Rica \\ mariadelcarmen.acuna@ucr.ac.cr
}

\section{Recibido: 1 abril 2013 Aceptado: 26 junio 2014 Corregido: 30 junio 2014}

\begin{abstract}
Resumen: Este es un ensayo teórico-reflexivo que pretende responder a la pregunta qué papel ha tenido la educación superior en la reproducción de prácticas de desigualdad entre los géneros. Para ello, dado que es un trabajo teórico-reflexivo, se presentan ideas construidas por la autora a partir de revisión bibliográfica y de sus propias reflexiones, derivadas del conocimiento y la experiencia adquiridos en los ámbitos académico y de activismo feminista. El ensayo se desarrolla en tres apartados. El primero, Educación superior y desigualdad de género, es una exposición del tema siguiendo perspectiva social, donde se abordan reflexiones como el ordenamiento de la sociedad en categorías binarias de género y la situación de las mujeres en la ciencia y las universidades. En el segundo apartado, Aproximaciones para entender el vínculo Género-Educación Superior, se brindan aclaraciones teóricas básicas en torno al enfoque de género y se mencionan algunas perspectivas desde las cuales se puede analizar la desigualdad de género en el nivel terciario de la educación; también, se hace una breve referencia a los procesos de diseño curricular y las intencionalidades presentes en las universidades. El tercer apartado, Currículum universitario, políticas públicas y cambio social, versa sobre la importancia de la educación superior para las sociedades, haciendo énfasis en la construcción de políticas públicas y en la generación de cambios sociales.Como conclusión, se afirma que es posible analizar el vínculo género-educación superior a partir de referentes teóricos e investigaciones existentes. La desventaja de las mujeres en el ámbito universitario es una realidad que requiere un cambio sociocultural y estructural; para lograrlo, es necesario desarrollar estrategias y acciones conjuntas entre diversos actores sociales, orientadas a impulsar prácticas igualitarias y equitativas entre los géneros dentro de la academia.
\end{abstract}

Palabras clave: Género, Educación Superior, Currículo, Políticas Públicas.

\begin{abstract}
This is a theoretical and reflexive essay that expects to answer the question which role does the Higher Education has had in the dissemination of gender inequalities. To achieve this, three sections are developed. The first section, explores Higher Education and gender inequality according to a social perspective, where considerations such as how society is arranged in gender binary categories, and women's situation in science and universities are addressed. The second section, Approaches for
\end{abstract}

\footnotetext{
1 Licenciada en Psicología de la Universidad de Costa Rica. Actualmente labora como Docente y Asesora en el Centro de Evaluación Académica, la Vicerrectoría de Acción Social y la Escuela de Formación Docente, todos de la Universidad de Costa Rica. En el Centro de Evaluación Académica, sus principales funciones son asesorar y acompañar procesos de evaluación, diseño curricular, innovación docente y seguimiento a carreras acreditadas. En la Vicerrectoría de Acción Social, sus principales funciones son analizar y asesorar proyectos de extensión docente. En la Escuela de Formación Docente, es profesora del curso Educación Sexual. Además, se desempeña como Profesora Invitada en varios cursos del Área de Salud. Recientemente ha publicado artículos junto con colegas docentes en la revista Actualidades Investigativas en Educación y en la revista Intersedes; ha sido conferencista en distintas actividades académicas, nacionales e internacionales.
} 
understanding the Gender-Higher Education bond, gives basic theoretical explanations about the gender approach, and mentions some Useful perspectives in order to analyze gender inequality in the tertiary level of education; in addition, it offers a brief reference about curricular design processes and intentions at universities. The third section, University curriculum, public policies and social change, refers to the importance Higher Education has in society, highlighting the development of public policies and the generation of social changes. As a conclusion, it affirms that it is possible to analyze the Gender-Higher Education bond upon theoretical references and available research. Women's disadvantage in University is a reality that requires a social-cultural and structural change; to reach $i t$, it is necessary to develop joint strategies and actions among diverse social agents, oriented to promote fair and equal actions between genders inside academy.

Keywords: Gender, Higher Education, Curriculum, Public Policies

\section{Introducción}

La organización patriarcal de la sociedad ha dominado todas las esferas de la vida humana; es posible identificar diferencias sociales debido al género en todos los ámbitos. La educación superior no ha sido una excepción. En este artículo se pretende analizar el vínculo entre la educación superior y las diferencias de género, respondiendo dos interrogantes: ¿Qué papel ha tenido la educación superior en la reproducción de prácticas de desigualdad entre los géneros? ¿Cómo puede contribuir la educación superior a promover la equidad entre los géneros?

En el primer apartado, se exponen reflexiones teóricas y personales desde una perspectiva social, a modo de exposición del tema. En el segundo apartado, se mencionan algunas posturas para entender el vínculo género-educación superior, desde el entendimiento de la finalidad de la educación (especialmente la universitaria) y desde aclaraciones conceptuales respecto al enfoque de género. Por último, en el tercer apartado, se analiza el papel de las universidades para el cambio social, haciendo referencia también a políticas públicas.

\section{Educación superior y desigualdad de género}

"En la educación formal estamos enseñando para el futuro, en el cual participarán ambos sexos, y si trabajamos para ello, tenderemos hacia una sociedad mejor, en la cual se aprovecharán las potencialidades de cada uno"

(González, 1992, p. 19)

Históricamente, la organización patriarcal de la sociedad ha dominado casi todas las esferas de la vida humana: "Los hombres, en tanto hombres, han dominado la historia, la política y la cultura" (Schmidt, 1992, p. 6). En cualquier espacio es posible identificar diferenciaciones sociales debido al género.

Ahora bien, ¿qué es género? La mayoría de las personas (con excepción de las intersexuales) nacen con una estructura corporal biológica específica de hembra o de macho, 
a partir de la cual a cada individuo socialmente se le asigna un lugar de mujer u hombre respectivamente y, con base en ello, las características, comportamientos y actitudes que se esperan son diferenciales para unas y para otros, de acuerdo con el sexo al que pertenece. Es decir, el género lo constituyen los aspectos psicológicos, sociales y culturales que se le adjudican a cada persona según sea su sexo (Campos y Salas, 2002).

En ese sentido, el ordenamiento de la sociedad se ha dado siguiendo categorías binarias: si se nace en un cuerpo de hembra, se es mujer y se enmarca dentro del género femenino; si se nace en un cuerpo de macho, se es hombre y se enmarca dentro del género masculino. De tal manera, estos binarismos genéricos es posible encontrarlos en cualquier espacio que determina la vida de las personas, en tanto funcionan como mandatos a seguir dentro del sistema patriarcal hegemónico que organiza la mayoría de las sociedades. Este funcionamiento dicotómico, que encasilla y restringe el marco de acción tanto de hombres como de mujeres, es necesario cambiarlo y, considerando que el género ha sido construido socialmente, es "susceptible de transformación y de renegociación" (Bertozzi, 1998, p. 6). Claro ejemplo de esto son las personas transgénero, transexuales e intersexuales, quienes desbordan y reconstruyen los límites establecidos para los cuerpos y para los géneros.

En los espacios educativos formales se ha desarrollado la ciencia y el conocimiento socialmente validado. Sin embargo, hasta el siglo XIX, la educación como institución social y el ámbito profesional-laboral fueron espacios predominantemente ocupados por hombres, donde este sistema patriarcal hegemónico se ha expresado, por ejemplo, mediante el cientificismo y racionalidad técnica. Esto es, ha prevalecido una visión androcéntrica en la ciencia, que ha sido parcializada y excluyente de otras visiones; es decir, de otras formas de conocimiento y de aproximación a las realidades. En ese sentido, "lo femenino ha sido devaluado de modo consistente en el sistema educativo (...) la producción cultural de la mujer se ha silenciado, trivializado o distorsionado en la construcción del saber que han hecho los hombres" (López, 1992, p. 4).

Específicamente en la educación superior, al menos en el mundo occidental, la influencia de lo masculino se encuentra en las metodologías, los procedimientos, los temas seleccionados y el lenguaje utilizado (González, 1992; Gore, 1996). La mirada de las mujeres ha estado relegada, teniendo como consecuencia una perspectiva parcializada en torno a los objetos de estudio y a las formas de acceder a ellos; por ejemplo: la intuición y los afectos, que son considerados culturalmente características femeninas, en los procesos investigativos no son reconocidos como elementos científicos. Inclusive, se propone la ciencia en sí misma como una actividad de dominio o invasión sobre los objetos de estudio, lo cual es una forma de aproximación falocéntrica; por ejemplo, es común que para estudiar algún fenómeno, se tomen elementos aislados del contexto, se induzcan situaciones (como en el caso de estudios experimentales) e, inclusive, se utilicen animales para la experimentación. Por el contrario, en sociedades pre-patriarcales, la aproximación de las mujeres al conocimiento y a los objetos de estudio era mediante relaciones de reciprocidad (Beirute, Chacón, Fonseca, Garita y Solano, 2007).

A lo anterior se une que el acceso a la educación formal no ha sido equitativo para ambos géneros, pues durante siglos (según los registros históricos, desde Grecia antigua hasta el siglo XIX) las mujeres han estado excluidas de ella, sobre todo a nivel universitario, y han debido dar grandes luchas para incorporarse en las instituciones de educación superior. Ahora 
bien, a partir del siglo pasado se han desarrollado importantes cambios sociales, políticos y económicos, que han impactado también la academia; entre estos, el ingreso de mujeres a la educación superior y el estudio de nuevos tópicos que antes no se abordaban, como es el caso de los estudios de género (Lau y Cruz, 2005).

En la actualidad, existe una mayor cantidad de mujeres estudiando, especialmente con edades inferiores a 25 años. Para el caso de Costa Rica, por ejemplo, a nivel de posgrado, la cantidad de mujeres estudiantes aumentó de un 44\% en el año 2000 al 50\% en el año 2009 (Programa Estado de la Nación, 2011). No obstante, pese a que las mujeres han accedido a los estudios universitarios y que la proporción de hombres y mujeres estudiantes es similar, las oportunidades de crecimiento profesional y académico de las mujeres son muy limitadas en comparación con los hombres (González, 1992; Lau y Cruz, 2005; Zapata, 2011). En el desempeño académico, el sexo es un factor de gran incidencia. Aún cuando las mujeres enfrentan condiciones más adversas para estudiar (tales como: doble o triple jornada de estudio y trabajo, quehaceres domésticos, crianza de hijas e hijos u otros familiares), se gradúan en mayor cantidad que los hombres: en el 2009, de los títulos otorgados, casi 63\% eran de mujeres (Programa Estado de la Nación, 2011). Además, según un estudio sobre tiempo de graduación en la Universidad de Costa Rica (Gallardo, Molina y Cordero, 2012, comunicación personal), se encontró que las mujeres se gradúan en menor tiempo que los hombres; esto concuerda con el Informe VII CEDAW (República de Costa Rica, 2008) donde se indica que las mujeres presentan mayor aprobación de cursos y menor repitencia en los niveles educativos que los hombres.

Vinculado con el tema de la educación en Costa Rica, en el Informe Sombra para la Convención sobre la Eliminación de todas las formas de Discriminación contra la Mujer de los años 2007 a 2010 (Agenda Política de Mujeres, 2010), se menciona que:

- El sexismo y la discriminación siguen extendidos en el sistema educativo.

- Pocas mujeres han ingresado a la educación técnica y al ámbito de la ciencia y tecnología.

- Persisten brechas de género en el mercado laboral debido a la falta de estímulo para ingresar en campos tradicionalmente masculinos.

- Mujeres reciben salarios menores que los hombres, por el mismo tipo de trabajo.

- Mujeres en trabajos que tradicionalmente han sido masculinos, son sujeto de discriminación y de reducidas oportunidades de acenso laboral.

- Pocas mujeres se encuentran en puestos de toma de decisiones dentro de su campo de trabajo.

Dentro de las estructuras universitarias, para el año 1992, se afirmaba que existen menos mujeres con grados académicos altos y en puestos de poder o de toma de decisiones (González, 1992). Casi una década después, para el año 2011, se sigue afirmando lo mismo: "cuando el nivel del título es mayor, la participación de las mujeres es menor" (Zapata, 2011, p. 17); de modo similar, la participación de las mujeres disminuye a medida que aumenta el nivel de los puestos académicos dentro de las jerarquías universitarias (Zapata, 2011). Esto se debe en parte a que muchas mujeres deben escoger entre seguir su carrera profesional o formar 
una familia (Lau y Cruz, 2005). Otros factores que determinan el ingreso, la permanencia y la movilidad de las mujeres en la educación superior son:

Los estereotipos dominantes frente a estudiantes y científicas que forman parte del sistema académico; la asociación de la carrera universitaria al modelo de las biografías masculinas, que se apoya en la correspondiente división del trabajo e ignora el problema estructural de la conciliación de la familia con el trabajo científico; la estructura del mercado de trabajo que ofrece menos oportunidades a las mujeres y un número mayor de inseguridades en el ejercicio de la profesión; la presión que exige a los científicos y las científicas una gran flexibilidad y una entrega absoluta a la profesión para poder avanzar en las estructuras jerárquicas del campo científico. (Zapata, 2011, p. 18-19).

Lo anterior significa que la discriminación hacia las mujeres está aún presente en las universidades, encontrando inclusive el sexismo académico como forma específica de inequidad en la educación superior. Aun cuando se ha demostrado abiertamente la existencia de discriminación por género, las instituciones universitarias no han asumido que esta problemática también se da dentro de ellas, en tanto se omite de "los análisis curriculares, características del estudiantado y del cuerpo docente y de las metodologías educativas y de investigación" (González, 1992, p. 9). Evidencia de ello es la carencia de datos desagregados por sexo, especialmente en torno a indicadores universitarios importantes, tales como: datos de matrícula, rendimiento académico (aprobación y reprobación de cursos), distribución de estudiantes por carrera, distribución de docentes, distribución de puestos de autoridad y de toma de decisiones; lo mismo sucede con características sociodemográficas que componen un perfil del estudiantado universitario, pues no hay cruce de variables con respecto al género.

Respecto a la toma de conciencia en torno a la inequidad que se vive en las universidades, ha habido etapas desde la negación del tema y de la discriminación, hasta la integración de cambios sociales en la vida académica (González, 1992). En ese sentido, cobra importancia revisar las prácticas cotidianas que se viven en las universidades y promover procesos de reflexión, tanto en torno a la educación superior en sí, como en cuanto a las profesiones que se aprenden en ella. Por citar solo un ejemplo, "la segregación en carreras por motivos de género" (República de Costa Rica, 2008, p. 33), en el sentido que en ciertas hay menor cantidad de mujeres, tales como las vinculadas con tecnología, pues tradicionalmente han sido consideradas profesiones masculinas.

Ahora bien, este análisis ya no solo es cuestión del currículum oficial-formal (es decir, de lo que explícitamente se enseña), sino que toma particular relevancia el currículum tácito u oculto, entendido como "aquellos aspectos que no están explícitos en la educación y, por tanto, no se es consciente de ellos, no se cuestionan" (Álvarez, 1995, p. 52). Es decir, el currículo oficial-formal son las propuestas educativas que están establecidas explícitamente en la planificación curricular; mientras que el currículo oculto son las dinámicas propias de la puesta en práctica de un proyecto curricular, las cuales se dan de manera implícita y, en ocasiones, inconsciente. Entonces, el currículum tácito u oculto comprende "valores, normas, actitudes, sentimientos, costumbres (...) que se expresan en un determinado uso de 
los espacios, los tiempos, el lenguaje, las relaciones y las estructuras organizativas" (Álvarez, 1995, p. 53). Al enlazar estos elementos con el tema de género (es decir, con la reproducción de roles diferenciados), se obtiene que:

- normas y valores, respecto a maneras de vivir y convivir, se presentan de forma diferenciada para hombres y mujeres;

- actitudes y conductas son permitidas y esperadas según el género, teniendo como referente las normas y valores;

- sentimientos, entendidos como "expresiones personales antes los acontecimientos que se producen a nuestro alrededor" (Álvarez, 1995, p. 54), son demostrados o reprimidos de acuerdo con convenciones sociales relacionadas al ser hombre o ser mujer;

- el lenguaje y la cultura, reproducen el modelo patriarcal hegemónico (Álvarez, 1995).

A partir de lo anterior, es posible señalar que a través del currículo oculto también se perpetúan prácticas sexistas en el ámbito educativo y, por lo tanto, es necesario analizarlo: "el sistema educativo será uno de estos sistemas de sedimentación del sexismo si el profesorado no pone especial atención en hacer cuestionar al alumnado su reproducción de los estereotipos y si no se cuestiona también su propio comportamiento en relación con el alumnado" (Artal, 2009 , p. 11). Cabe aclarar que cuando se menciona el sexismo en la educación (o bien, la educación sexista), se hace referencia a la "enseñanza de la masculinidad o de la femineidad, a partir de prácticas culturales heredadas y que promueven valores determinados y opuestos" (Bertozzi, 1998, p. 19); es decir, cuando se educa desde una concepción donde se otorgan atributos específicos a los hombres o a las mujeres, con base en su sexo.

De los citados elementos que conforman el currículo oculto, se retoma el lenguaje, a modo de ejemplo. Este es una institución social, en tanto se trata de "un sistema de comunicación que estructura nuestra percepción de la realidad" (Schmidt, 1992, p.1). En ese sentido, el lenguaje es una forma de poder, que también se ha utilizado para excluir a las mujeres (López, 1992). Mediante la lengua y sus usos, se crean "valores, actitudes y concepciones del mundo... La adquisición del lenguaje significa adquirir también los modelos de comportamiento, creencias, pensamientos y emociones que él confiere" (Schmidt, 1992, p. 1-2).

Por lo tanto, siguiendo el ejemplo y yendo a lo concreto, en los procesos de análisis de la educación superior y de cambio de prácticas sexistas, uno de los primeros pasos es modificar el lenguaje que se utiliza, en tanto este es "la cristalización de la cultura propia del sistema social imperante" (Álvarez, 1995, p. 54). Las formas de expresión articulan, definen y revelan la visión de mundo que tienen las personas; es decir, el lenguaje es estructurante de la percepción que se tiene en torno a la realidad y, de ese modo, construye realidades, de ahí la importancia de modificar la forma de hablar y de escribir, pues conllevan una manera de entender el mundo.

Pasando a un plano de análisis más general, en la educación superior se debería promover un currículo balanceado, entendido como aquel que "busca la integración de grupos y sectores que han sido excluidos de la academia, tradicionalmente dominada por un currículo exclusivo que presenta al hombre como centro y como norma, y la experiencia masculina como la universal" (Schmidt, 1992, p. 5). Volviendo al lenguaje, en él se encuentran manifestaciones 
del sexismo que implican un desbalance en la equidad entre los géneros, a saber: "el uso del masculino genérico, el léxico, la morfología, los refranes, las expresiones idiomáticas, la representación en general de la mujer" (Schmidt, 1992, p. 2). En la misma línea, se propone desarrollar un currículo género sensitivo e inclusivo (Bolaños, 2003), en el sentido de generar proyectos educativos universitarios balanceados en materia de género y de incorporar la mirada femenina del mundo en los procesos educativos formales.

Lo anterior implica analizar "la realidad social en la que está inmersa una carrera, una disciplina, una profesión, la universidad y el país" (Bolaños, 2003, p. 74). En la actualidad, las universidades enfrentan retos sociales importantes, dentro de los cuales es preciso resaltar "la inclusión y movilidad social justa para todos los sexos en todos los niveles y ámbitos de los espacios educativos" (Zapata, 2011, p. 6). Esto se logra, por una parte, mediante la revisión colectiva y consciente de los marcos referenciales que sustenta una carrera, es decir, los fundamentos socio-históricos, epistemológicos y pedagógicos de una disciplina; por otra parte, a través de la promulgación de políticas universitarias y del trabajo de sensibilización con los sectores poblaciones que integran una comunidad universitaria: estudiantado, cuerpo docente, personal administrativo y autoridades institucionales (esto se abordará más adelante, en el apartado Currículum universitario, políticas públicas y cambio social).

Para entender el vínculo entre el género y la educación superior es preciso referirse a algunas aristas, en este caso se abordan tres: la intencionalidad de las propuestas educativas, el enfoque de género y la finalidad de introducir la perspectiva de género en la educación superior.

\section{Aproximaciones para entender el vínculo Género-Educación Superior}

"Educar para la democracia obliga a incluir la perspectiva de género en la educación" (Artal, 2009, p. 13)

Una de las principales instancias socializadoras es la escuela como institución; es decir, la escuela entendida como todos los niveles de la educación formal, desde el preescolar hasta la universidad. Como se ha venido señalando, la educación tiene un papel central en la reproducción de los estereotipos de género:

En el proceso de socialización en el que todas las personas estamos inmersas, la escuela, la familia, y los medios de comunicación aparecen como las tres grandes instituciones que influyen en mayor grado en la identidad humana y en la clasificación social de los individuos en función del sexo al que se adscriben, convirtiéndose la diferencia biológica en una diferencia social. (Artal, 2009, p. 10).

Como se verá más adelante, los procesos educativos tienen una intencionalidad, en tanto toda propuesta curricular responde a los intereses de determinados grupos y personas con 
visiones de mundo específicas. Esto significa que los planes de estudio universitarios tienen un propósito claramente establecido; es decir, están planteados desde un para qué: ¿Para qué preparamos a los profesionales y las profesionales? ¿Quién o quiénes se benefician con dicha formación? ¿Qué se impacta con dicha formación?” (Bolaños, Vargas y Velázquez, 1999, p. 186). Y esto indistintamente de los procesos de diseño curricular que se haya desarrollado y de los niveles de participación que hayan tenido en ellos los distintos sectores interesados.

Ahora bien, en la puesta en práctica del currículum, este es implementado por las y los docentes desde posturas epistemológicas personales en torno a diversos componentes: los contenidos que se enseñan, el plan de estudios del cual se forma parte, la docencia en sí misma (y, en particular, la docencia universitaria) y la finalidad de la educación superior. Con esto se observa entonces que la intencionalidad propia de cada persona docente, sea esta consciente o no, y explícita o no, también es parte los procesos de formación de profesionales.

Por lo tanto, a partir de los dos anteriores es posible decir que la educación superior tiene varias finalidades según los distintos actores vinculados a ella y las posturas epistemológicas que poseen (esto sin considerar si tienen o no claridad respecto a ellas): "el plan de estudios prescrito por una institución acota el trabajo docente pero, simultáneamente, es traducido y resignificado en la práctica por los/as profesores/as que poseen identidades disciplinarias o institucionales específicas" (Cardaci, 2005, p. 137).

Una de estas perspectivas que orientan la labor universitaria, el ejercicio profesional y la vida en general, es justamente el enfoque de género. Por consiguiente, una explicación que cabe retomar en este texto es la de perspectiva de género, la cual es entendida como una mirada particular para comprender la vida social:

A partir de la idea de que ser hombre o ser mujer es un dato cultural y no biológico, y que la forma que adoptan las desigualdades sociales basadas en el sexo de las personas se relaciona con la manera como se construye la oposición hombre/mujer en el imaginario social. (Palomar, 2005, p. 18).

Otros dos enfoques comúnmente utilizados que es preciso aclarar, para entenderlos y diferenciarlos, son los Estudios de Género y los Estudios de la Mujer; si bien están estrechamente relacionados e intersecados, se diferencian en cuanto a sus objetos de estudio.

Los Estudios de Género (EG, por sus siglas) parten de la premisa de que los conceptos de mujer y de hombre no son hechos naturales, sino construcciones culturales; por ende, su objeto de estudio son "las relaciones socioculturales entre mujeres y hombres (hombres y hombres/mujeres y mujeres)" (González, 2009, p. 682). Los Estudios de la Mujer (EM, por sus siglas) tienen como objeto de estudio las mujeres (González, 2009). Ambos se fundamentan en las teorías feministas y tuvieron origen en instituciones de educación superior de países industrializados: los Estudios de la Mujer en los años 1970 y los Estudios de Género en la década de 1980; por ende, los EM son antecedente de los EG. Por ende, a partir de dichos objetos de estudio, se podría decir que los EG tienen un alcance más amplio.

Además, se plantea que es necesario ubicar categorías para el análisis de género en 
los espacios donde estas se manifiestan: los roles de género, en la esfera doméstica; los estereotipos de género, en la esfera laboral y las asimetrías de género, en ambos espacios, laboral y doméstico (Fernández, 2010).

Cuando se realizan investigaciones en torno a las diferencias entre mujeres y hombres (relativas, por ejemplo, a conductas, actitudes y aptitudes), desde perspectivas biológicas se explican los resultados en términos corporales, tales como: genes, hormonas, estructuras cerebrales. Por su parte, desde la perspectiva de género, las diferencias se explican en términos socioantropológicos, como producto de la educación y los procesos sociales. Por lo tanto, en el sentido de analizar las finalidades y entender las miradas de la investigación, los EG plantean preguntas en torno a los procesos investigativos en sí mismos: "¿quiénes y con qué propósitos argumentan acerca de la diferencia sexual?; ¿cómo se construyó el significado?; ¿qué cualidades o aspectos se consideran?” (González, 2009, p. 683).

En esa línea, García (2005) expone varias perspectivas para entender el fenómeno de la desigualdad de género y su vínculo con la educación como institución social. Si bien el análisis lo plantea para el ámbito de la escuela primaria, son aproximaciones aplicables también al tercer nivel de la educación (es decir, el ámbito universitario).

- Perspectiva del feminismo liberal. Mediante la socialización, la escuela genera prácticas discriminatorias: "el sistema escolar es el responsable de instalar entre las y los infantes actitudes sexistas (García, 2005, p. 71). La desigualdad es causada por ignorancia, prejuicios y falta de modelos a seguir, pues en los textos escolares las figuras que aparecen por lo general son hombres; por lo tanto, es preciso "re-trabajar el actual sistema escolar para modificarlo sin conflictos. Concienciar y remover los sesgos en la educación son la respuesta" (García, 2005, p. 71). Es decir, hay carencia en la diversidad de figuras de identificación que se ofrecen en el sistema escolar.

- Perspectiva radical. El origen de la diferencia está en las estructuras de poder: la escuela es el "espacio de transmisión del conocimiento masculino", en el sentido que el sistema educativo muestra únicamente la experiencia masculina, lo cual es "una herramienta que mantiene el dominio masculino y/o el patriarcado" (García, 2005, p. 72).

- Perspectiva marxista o socialista. La escuela y otras instituciones reproducen "relaciones de desigualdad entre los géneros y las clases sociales"; a partir de la explicación de la reproducción social, pretende responder al "cómo se reproduce el género" (García, 2005, p. 73). Se afirma que existe una "estrecha relación entre el sistema educativo y la división sexual del trabajo", en el sentido que "ambas instituciones son funcionales al sistema capitalista en la medida que contribuyen a la producción y reproducción de la fuerza laboral" (García, 2005, p. 73). Esta es entonces una explicación compleja que une la desigualdad de género con la desigualdad de clase.

- Perspectiva del feminismo posmodernista. "Las niñas y jóvenes son entes complejos con múltiples identidades, subjetividades y discursos de género, los cuales no necesariamente siguen o se apropian de los discursos alternativos (feministas)" (García, 2005, p. 74). Es decir, si por ejemplo se les muestran historias feministas, no las entienden porque ya han sido moldeadas con cuentos infantiles tradicionales que reproducen el discurso regulador dominante. 
Por lo tanto, desde el enfoque de género se señala que "el género -en tanto pregunta acerca de cómo se interpreta la diferencia sexual- atraviesa prácticamente todo el campo educativo" (González, 2009, p. 692). De tal manera, se afirma que el conocimiento científico no es neutral, pues en la producción de conocimiento ha prevalecido la perspectiva de quienes poseen más recursos y ostentan el poder (el cual puede ser económico y político). Se buscan entonces alternativas al saber que se ha instituido desde los espacios y grupos de poder. Cabe recordar que en los procesos de diseño curricular, se dan luchas de poder entre grupos que buscan incluir sus intereses o demandas dentro de los planes de formación de profesionales; es decir, se dan dinámicas de "negociación/imposición de las diversas visiones de mundo en un contexto determinado, para concretar una propuesta curricular que tenga posibilidades de logro" (Bolaños, Vargas y Velázquez, 1999, p. 186).

En este punto, entra la pregunta del para qué el enfoque de género en la educación superior, cuyas respuestas pueden ser múltiples; con este artículo se proponen algunas:

- para analizar las disciplinas y sus prácticas, las formas de acceder al conocimiento que las caracterizan y de enseñarlo, orientando dicho análisis al entendimiento de cómo se reproducen estereotipos de género;

- para reflexionar cómo desde cada carrera se puede contribuir a promover la equidad entre los géneros e impulsar estrategias y acciones en ese sentido;

- para reducir las brechas entre hombres y mujeres tanto en el espacio laboral como en el desarrollo profesional-académico;

- para contribuir desde la educación superior y las profesiones a mejorar condiciones de vida de las mujeres.

Vinculado con ello, respecto al análisis de la labor docente, Cardaci (2005, p. 138) menciona que se deben cuestionar diversos aspectos: "las creencias, los contenidos y las estrategias didácticas que se utilizan en el aula, así como las apreciaciones, los estereotipos y las actitudes personales de los/as profesores/as hacia la enseñanza de determinados asuntos y problemas". En esta tarea, que no es pequeña, es imprescindible trabajar con las y los docentes, pues son quienes tienen la mayor responsabilidad en la implementación de una propuesta curricular.

En síntesis, es necesario "desarrollar una pedagogía más progresista y programas que contrarresten las desigualdades" (García, 2005, p. 75-76). Por lo tanto, la labor de las universidades es vital para el cambio en las dinámicas sociales, lo cual es objeto del siguiente apartado.

\section{Currículum universitario, políticas públicas y cambio social}

"la educación es uno de los pilares fundamentales de los derechos humanos, la democracia, el desarrollo sostenible y la paz, por lo que deberá ser accesible para todos a lo largo de toda la vida"

(UNESCO, 1998, párr. 9) 
Para retomar el concepto central de este artículo, el género, cabe señalar que este es entendido como "principio ordenador de las relaciones sociales basado en la diferencia sexual, que produce distintos efectos tales como jerarquías, distinciones y categorías diferenciales para las personas" (Palomar, 2005, p. 7). Es decir, el concepto de género sirve también como categoría de análisis para "entender adecuadamente las relaciones asimétricas entre mujeres y varones" (Fernández, 2010, p. 256). De estos aportes se extrae que el género y el poder están relacionados, en tanto ambos participan en el proceso organizador de las relaciones sociales (Palomar, 2005); en ese sentido, es innegable que en las sociedades patriarcales, la desigualdad entre mujeres y hombres tiene claramente un carácter político, pues se relaciona con el acceso al poder: "la subordinación social y económica de las mujeres tiene un carácter político", debido a que expresa la "relación de desigualdad entre los géneros" (García, 2005, p. 77).

Vale aclarar que los términos sexo y género son conceptos distintos, cuyo uso no necesariamente ha conllevado a reflexiones adecuadas. Fernández (2010) explica que son dominios científicos diferentes: "el sexo hace referencia a lo biológico y el género a lo social (...) lo biológico y lo social se complementan a la hora de formar una única, aunque compleja, realidad" (p. 257); es decir, ambos conceptos deben leerse como complementarios. Inclusive, desde la filología, el género se utiliza gramaticalmente aplicado a las palabras, mientras que el sexo se refiere "a la condición sexuada de las personas" (Fernández, 2010, p. 259).

Ahora bien, el uso de ciertos términos, que son transformados en conceptos, es un acto político que responde a ideologías determinadas. Es decir, los significados y los usos de términos como género, equidad de género e igualdad de género, varían dependiendo del actor social que los utiliza, tales como "políticos(as), académicas(os), activistas sociales o ciudadanos" (Rosales y Flores, 2009, p. 68).

Yendo al ámbito de la educación, es preciso entender que el currículum es una propuesta político-educativa, cuya formulación está permeada por negociaciones y luchas entre diversos grupos, quienes pretenden plasmar sus ideologías, necesidades e intenciones y con ello orientar los procesos de formación de personas. En ese sentido, el currículum universitario puede considerarse "una propuesta político-educativa, producto de una construcción cultural de tipo sistémico, realizada por y para la formación de personas y vivenciada en un ámbito pedagógico, en el cual interactúan diversas visiones de mundo" (Equipo de Diseño Curricular, 2009, p. 1). Las políticas públicas permean los diseños curriculares y las acciones que se desarrollan en las instituciones de educación superior, pues dictan lineamientos que deben ser cumplidos. Además, las demandas del Estado son una de las fuerzas que inciden en la configuración del currículum universitario y su puesta en práctica.

De la misma manera, en la formulación de las políticas públicas (incluyendo las políticas educativas), pueden incidir distintas fuentes: "los conocimientos emanados de la investigación académica, las demandas ciudadanas de ciertos organismos de la sociedad civil y los actores gubernamentales" (Rosales y Flores, 2009, p. 69). En el tema de género, se visualizan tres actores: el Estado (con sus instituciones gubernamentales), la sociedad civil (en este caso, organizaciones y activistas feministas particularmente) y las universidades (en especial, las públicas). Vianna y Unbehaum (2006) señalan que la tarea de consolidar el enfoque de género en las políticas educativas le corresponde al Estado; sin embargo, son los grupos e individuos 
de la sociedad civil quienes en su mayoría intentan llevar la agenda de género a las políticas nacionales, con el fin de incidir en las estructuras de poder y en las prácticas socioeconómicas que perpetúan la desigualdad entre los géneros.

En la construcción de las políticas públicas, un aspecto primordial es tomar como respaldo elementos legales propios de la normativa del país y del marco jurídico internacional. Vale acotar que la mayoría de instrumentos internacionales se inscriben en el feminismo de la igualdad, pues pretenden "realzar el lugar de la mujer y luchar porque estas obtengan iguales derechos que los hombres" (Muñoz, 2006, p. 394). Por otra parte, no se debe olvidar que las políticas nacionales fungen como referente para los procesos educativos en todos los niveles y, que cada institución debe ponerlas en práctica a partir de sus propias características y su propia cultura.

El aporte de las universidades como actores decisivos en la formulación de política pública, consiste en que:

Los resultados de las investigaciones tienen una función y utilidad social. En las universidades la investigación es una actividad sistemática de análisis que no sólo reporta datos, sino que éstos pueden apoyar la política de planeación y la toma de decisiones (García, 2005, p. 76).

Siguiendo a Rosales y Flores (2009), es posible afirmar que el espacio académico (es decir, las universidades) es parte de las arenas que conforman la política social. Se propone entonces a la academia como actor social, debido a la producción de conocimiento que se da en las instituciones de educación superior, la cual sirve como referente para elaborar políticas. Es decir, las universidades son espacios públicos donde se generan "conocimientos científicos que ayudan a comprender y afrontar los problemas sociales y su responsabilidad de promover el respeto por la diversidad, la tolerancia y las diferencias de género de las personas" (Palomar, 2005, p. 10).

Ahora bien, además de la incidencia en la construcción de políticas públicas, las universidades desempeñan un papel central en la sociedad y en los cambios culturales. La UNESCO (1998a) visualiza la importancia y la labor de la educación superior para el desarrollo de los países y el cambio social. Además, afirma que en el siglo XXI ha habido una mayor toma de conciencia respecto la importancia de la educación superior "para el desarrollo sociocultural y económico y para la construcción del futuro" (UNESCO, 1998a, párr. 1).

La educación superior tiene gran relevancia en el impulso de prácticas igualitarias y equitativas entre los géneros. Como bien indican Montesinos y Romero (2010), la educación y, especialmente, la educación superior, permite cambiar la condición social de las personas. De igual manera, la educación superior durante siglos ha demostrado que es capaz de "transformarse y propiciar el cambio y el progreso de la sociedad" (UNESCO, 1998a, párr. 3).

Al hablar de educación superior, la UNESCO (1998a) define que esta comprende "todo tipo de estudios, de formación o de formación para la investigación en el nivel postsecundario, impartidos por una universidad u otros establecimientos de enseñanza que estén acreditados por las autoridades competentes del Estado como centros de enseñanza superior" (UNESCO, 1998a, párr. 1).

Volviendo al rol social de las universidades, estas instituciones tienen un llamado a ser 
"punta de lanza, conciencia lúcida y espacio generador de conocimientos y acciones concretas en el desafío por lograr una sociedad justa y equilibrada, donde la paz, la democracia, la soberanía y la libertad sean pilares de la vida" (González, 2006, p. 281). Si bien González (2006) plantea esto para las instituciones de educación superior públicas, también es extensivo a las privadas, por cuanto las universidades en general forman las personas profesionales de un país y, también, constituyen "la base de la innovación científica y tecnológica" (Programa Estado de la Nación, 2011, p. 178). Es decir, se apela a que la educación superior contribuya en gran medida con el desarrollo de una nación, en los ámbitos intelectual-filosófico, científico, artístico-cultural, social y productivo.

Montesinos y Romero (2010) proponen que la educación superior es un vehículo emancipador, en tanto mueve a las personas en sus lugares dentro de la jerarquía social; es decir, sirve como medio para cambiar la condición social de las personas, dado que las aproxima a perspectivas críticas en torno al orden establecido y conduce a modificar las estructuras sociales. Según se explica, "la educación superior se constituye en la vía más eficiente para ofrecer a los individuos el acceso a la oportunidad de desarrollar habilidades y conocimientos que los sitúe en una mejor posición en la estructura jerárquica de sociedades diferenciadas" (Montesinos y Romero, 2010, p. 56). En el caso de las mujeres, con el acceso a la educación superior y la obtención de un título universitario, se ubican en una nueva posición de poder, en un nuevo estatus social, pues acceden al conocimiento (es decir, al poder intelectual) y a una profesión (conducente al poder económico), los cuales en la organización patriarcal de la sociedad han sido fuente de poder masculino. Cabe recordar que las mujeres se gradúan en mayor porcentaje que los hombres: 63\% las primeras y 37\% los segundos (Gutiérrez, Kikut, González y Alfaro, 2012). Por ende, se da un cambio de roles y, en ese sentido, se contribuye con el cambio cultural (Montesinos y Romero, 2010).

Para contribuir con ese cambio social, es necesario que al interior de las universidades se desarrollen acciones orientadas a impulsar la equidad de género en la educación superior. En efecto, el Marco de acción prioritaria para el cambio y el desarrollo de la educación superior promulgado por la UNESCO (1998b) establece algunas acciones relativas al tema de género; por ejemplo, se apela a que los Estados definan y apliquen "políticas destinadas a eliminar de la enseñanza superior todos los prejuicios fundados en el género y consolidar la participación de la mujer en todos los niveles y todas las disciplinas" (UNESCO, 1998, numeral 1, inciso i).

Lograr lo anterior implica "promover un cambio ético en las instituciones para que éstas incorporen en sus sistemas axiológicos el respeto a la diversidad y la búsqueda de la equidad, particularmente la equidad de género" (Palomar, 2005, p. 11). Entonces, para que las acciones intrauniversitarias en pro de la equidad tengan un impacto real, es necesario "implicar a todos los estamentos de la institución" (González, 2006, p. 273); en esta línea, el tema de género debería abordarse desde las tres actividades sustantivas: docencia, investigación y acción social; a manera de ejemplo:

Investigación, mediante la realización de proyectos concretos; docencia, con la incorporación de estas temáticas en los planes curriculares; acción social, a través de la prestación de servicios de prevención y atención de la violencia intrafamiliar contra las mujeres en Costa Rica (González, 2006, p. 272) 
Retomando las propuestas curriculares, cabe reconocer que el tema de género se ha incorporado como un contenido específico en ciertos cursos, como el tema central en otros o como eje transversal en planes de estudio. Para lograrlo, feministas académicas han realizado grandes esfuerzos, enfrentando barreras institucionales (como la burocracia en los procesos de rediseño curricular) y obstáculos externos: "las limitaciones sobrepasan los muros universitarios, ya que la ideología e idiosincrasia imperantes entre quienes diseñan políticas públicas y toman decisiones se reflejan no solo en la academia, sino en la sociedad en su conjunto" (Rosales y Flores, 2009, p. 73).

En cualquier espacio, la sensibilización es primordial para que las personas estén informadas y tomen consciencia de la necesidad de cambio. Como lo explica Hidalgo (2009), una estrategia es desarrollar talleres para que las personas interioricen la perspectiva de género, los cuales deberían orientarse a demostrar que "todos tenemos una práctica de género (...) si el género se hace, también se puede deshacer, es decir, se puede subvertir" (Hidalgo, 2009, p. 307).

Se podría afirmar que en las poblaciones que integran una comunidad universitaria (estudiantes, docentes, personal administrativo y autoridades universitarias) existen nociones en torno a la diferenciación entre los géneros, las cuales van desde un conocimiento básico de ciertos elementos hasta estudios avanzados en el tema. La tarea entonces es trabajar a profundidad los estereotipos existentes para borrarlos y, de esa manera, ir logrando paulatinamente la equidad entre los géneros en las instituciones universitarias.

\section{Conclusiones}

A partir de las reflexiones anteriores, se denota que existen investigaciones y referentes teóricos suficientes que permiten mirar la realidad del vínculo género-educación superior, a la luz de esos aportes.

Respecto a esta realidad, se requieren acciones y estrategias conjuntas que integren los distintos actores sociales para lograr un cambio que sea social-estructural, histórico y cultural: "La equidad e igualdad de géneros (...) exigen un compromiso de gestión de múltiples aspectos, algunos más complejos que otros, a fin de lograr avances que se buscan y establecer los correspondientes dispositivos de cumplimiento" (González, 2006, p. 273). Aunque quizá no con el ritmo deseado, es posible afirmar que las relaciones entre los géneros están siendo redefinidas como parte de "un cambio cultural que marca a la modernidad", el cual transforma los espacios públicos y privados (Montesinos y Romero, 2010, p. 57).

La importancia de incorporar la perspectiva de género en las universidades radica en que estas son espacios públicos donde se construyen conocimientos, se genera reflexión, se investiga en torno a problemáticas de la realidad y se desarrollan estrategias de acción social que inciden en el contexto.

Educar desde el enfoque de género es, en última instancia, educar para la democracia y para la convivencia pacífica en sociedad. Y, educar para la democracia "obliga a incluir la perspectiva de género en la educación” (Artal, 2009, p. 13). 


\section{Referencias bibliográficas}

Agenda Política de Mujeres. (2010). CEDAW 2007-2010 Costa Rica Shadow Report [Informe Sombra de Costa Rica para la CEDAW 2007-2010]. San José, Costa Rica: Agenda Política de Mujeres. Recuperado de http://www2.ohchr.org/english/bodies/cedaw/docs/ ngos/AgendaPol\%C3\%ADticadeMujeresforthesession en.pdf

Álvarez, C. (1995). El currículum oculto y sus manifestaciones. En Sara Acuña (Coord.), Coeducación y tiempo libre (pp. 48-68). Madrid, España: Popular.

Artal, M. (2009, junio). Construir el género: el cuestionamiento del sexismo y del androcentrismo en el sistema educativo. Acciones e Investigaciones Sociales 27, 5-21. España: Escuela Universitaria de Estudios Sociales, Universidad de Zaragoza.

Beirute, T., Chacón, M., Fonseca, A., Garita, N. y Solano, L. (2007). La naturalización de la diferencia: el vínculo ciencia y género. Revista Reflexiones 86-1, 9-27. Recuperado de http://www.latindex.ucr.ac.cr/reflexiones-86-1/reflexiones-86-1-01.pdf

Bertozzi, Y. (1998). Alfabetización en género para la vida cotidiana. San José, Costa Rica: Asociación Alfalit y Fundación para los Derechos Humanos de la Mujer.

Bolaños, C. (2003). Currículum universitario género sensitivo e inclusivo. Revista Ciencias Sociales, III-IV (101-102), 71-78.

Bolaños, C.; Vargas, A. y Velázquez, L. (1999). Hacia un diseño curricular alternativo en la educación superior. Educación, 23 (especial), 185-194. San José: Universidad de Costa Rica. Recuperado de http://www.cea.ucr.ac.cr/diea/documentos/Dise\%F10\%20 curricular\%20alternativo.pdf

Campos, Á. y Salas, J. M. (Comp.). (2002). Masculinidades en Centro América. San José, Costa Rica: Lara Segura Editores.

Cardaci, D. (2005). ¿Ausentes o invisibles? Contenidos sobre las mujeres y los géneros en el currículo de licenciatura de universidades mexicanas. La Ventana, Revista de Estudios de Género, (21), 107-142. México: Universidad de Guadalajara. Recuperado de EBSCOhost Academic Search Complete database.

Equipo de Diseño Curricular (2009). El currículo. Manuscrito inédito, Departamento de Investigación y Evaluación Académica, Centro de Evaluación Académica, Universidad de Costa Rica, San José, Costa Rica. 
Fernández, J. (2010). El sexo y el género: Dos dominios científicos diferentes que debieran ser clarificados. Psicothema, 22(2), 256-262. Recuperado de http://www.psicothema.com/pdf/3724.pdf

García, P. (2005). Género, educación y política pública. La Ventana, Revista de Estudios de Género, (21), 70-89. México: Universidad de Guadalajara. Recuperado de http://redalyc. uaemex.mx/pdf/884/88402103.pdf

González, M. (1992). Cuestionar lo incuestionable: el sexismo en el proceso educativo. En Proyecto de Estudios de la Mujer, Hacia un currículo no sexista: integración de los estudios de la mujer y el género en los cursos introductorios de español, inglés y ciencias sociales en la UPR (pp. 1-21). Cayey: Universidad de Puerto Rico.

González, R. M. (2009, julio-setiembre). Estudios de género en educación, una rápida mirada. Revista Mexicana de Investigación Educativa, 14(42), 681-699. México: Consejo Mexicano de Investigación Educativa. Recuperado de http://www.redalyc.org/articulo.oa?id=14011807002

González, Y. (2006). Equidad de género e inclusión social como tareas inconclusas de la Universidad de Costa Rica. En Educación y Universidad (pp. 271-281). (1a. ed.). San José, Costa Rica: Editorial UCR.

Gore, J. (1996). Pedagogías críticas y pedagogías feministas: ¿adversarias, aliadas u otra cosa? En Controversias entre las pedagogías: discursos críticos y feministas como regímenes de verdad (pp. 33-71). (2a. ed.). Madrid, España: Morata.

Gutiérrez, I., Kikut, L., González, R. y Alfaro, X. (2012). Seguimiento de la condición laboral de las personas graduadas 2000-2007 de las Universidades Costarricenses. San José, Costa Rica: Consejo Nacional de Rectores, Oficina de Planificación de la Educación Superior.

Hidalgo, A. (2009). Avances, observaciones y tareas pendientes: coloquio sobretransversalización de la perspectiva de género en la educación. La Ventana, Revista de Estudios de Género, (29), 305-309. Recuperado de http://www.redalyc.org/articulo.oa?id=88411884015

Lau, A. y Cruz, M. P. (2005). La incorporación de los estudios de mujeres y de género a las instituciones de educación superior. El Programa de Especialización-Maestría en Estudios de la Mujer de la UAM-Xochimilco. La Ventana, Revista de Estudios de Género (21), 228-251. Recuperado de http://www.redalyc.org/articulo.oa?id=88402108

López, I. (1992). El conocimiento sin exclusiones: la importancia de la revisión curricular. En Proyecto de Estudios de la Mujer, Hacia un currículo no sexista: integración de los estudios de la mujer y el género en los cursos introductorios de español, inglés y ciencias sociales en la UPR (p. 1-10). Cayey: Universidad de Puerto Rico. 
Montesinos, R. y Romero, R. (2010, mayo-junio). Democracia, educación y género: un reto social para el siglo XXI. El Cotidiano, Revista de la Realidad Mexicana, (161), 55-62. Recuperado de http://www.redalyc.org/articulo.oa?id=32513865008

Muñoz, F. (2006, mayo-agosto). Caminos cruzados: género en las políticas educativas en el Perú en los últimos diez años. Educação \& Sociedade, 27(95), 385-406. Brasil: Centro de Estudos Educação e Sociedade. doi: 10.1590/S0101-73302006000200004.

Palomar, C. (2005). La política de género en la educación superior. La ventana, Revista de Estudios de Género, (21), 7-43. Recuperado de http://148.202.18.157/sitios/publicacionesite/ pperiod/laventan/Ventana21/7-43.pdf

Programa Estado de la Nación en desarrollo humano sostenible. (2011). Tercer Informe de El Estado de la Educación. San José, Costa Rica: autor.

República de Costa Rica. (2008). Informe combinado de la Convención para la eliminación de todas las formas de discriminación contra la mujer - CEDAW, periodo de Marzo del 2003 a Abril del 2007 (Informe VII CEDAW). Recuperado de http://unpan1.un.org/intradoc/ groups/public/documents/icap/unpan049352.pdf

Rosales, A. y Flores, A. (2009, setiembre). Género y sexualidad en las universidades públicas mexicanas. Íconos, Revista de Ciencias Sociales, 35, 66-75. Quito, Ecuador: Facultad Latinoamericana de Ciencias Sociales, Sede Académica de Ecuador. Recuperado de EBSCOhost Academic Search Complete database.

Schmidt, A. (1992). Se puede hacer el milagro... o la compatibilidad de los objetivos de los cursos de Español Básico y la renovación curricular. En Proyecto de Estudios de la Mujer, Hacia un currículo no sexista: integración de los estudios de la mujer y el género en los cursos introductorios de español, inglés y ciencias sociales en la UPR (pp. 1-7). Cayey: Universidad de Puerto Rico.

UNESCO (1998a). Declaración mundial sobre la educación superior en el siglo XXI: visión $y$ acción. París, Francia: UNESCO. Recuperado de http://www.unesco.org/education/ educprog/wche/declaration spa.htm

UNESCO (1998b). Marco de acción prioritaria para el cambio y el desarrollo de la educación superior. París, Francia: UNESCO. Recuperado de http://www.unesco.org/education/ educprog/wche/declaration spa.htm 
Vianna, C. y Unbehaum, S. (2006, mayo-agosto). Gênero na educação básica: quem se importa? Uma análise de documentos de políticas públicas no Brasil. Revista Educação \& Sociedade, 27 (95), 407-428. Campinas, Brasil: Centro de Estudos Educação e Sociedade. Recuperado de http://www.scielo.br/pdf/es/v27n95/a05v2795.pdf

Zapata, M. (Junio, 2011). Movilidad e inclusión social: retos para la educación universitaria. Conferencia presentada en el I Congreso Internacional Universitario Géneros, Feminismos y Diversidades (GEFEDI), organizado por el Instituto de Estudios de la Mujer, Universidad Nacional de Costa Rica. Memoria electrónica. Recuperado de http:// www.gefedi.una.ac.cr/images/img gefedi/memoriagefedi2011.pdf 